schengerechter Arbeitsgestaltung (z. B. aus der ISO-EN-Norm 9241, Teil 2) vor dem Einsatz des Leiharbeiters nachweisen, dass er den Arbeitsplatz und die Arbeitsbedingungen entsprechend gestaltet hat.

\section{FAZIT}

Sowohl das Beispiel der Arbeitsbedingungen in der Leiharbeit wie auch die Auswirkungen von Restrukturierungen auf die Gesundheit der Beschäftigten zeigen, dass der im Arbeitsschutzgesetz formulierte, weitreichende Ansatz einer menschengerechten Arbeitsgestaltung dringend in der Arbeitsrealität ankom- men muss. Darauf verweist auch die europäische Expertengruppe HIRES (vgl. den Beitrag von Klaus Pickshaus in diesem Heft) mit ihrem expliziten Bezug zur Bedeutung der Gefährdungsbeurteilung, auch im Sinn einer prospektiven Strategie: „Um mögliche ungünstige Auswirkungen der Restrukturierung auf die Gesundheit und Sicherheit der Beschäftigten zu minimieren, sollte die Bewertung der Risiken sowohl vor, während, als auch nach der Restrukturierung erfolgen und anschließend in einem Maßnahmenprogramm zur Lösung der aufgedeckten Probleme umgesetzt werden". ${ }^{11}$ Die Restrukturierungsmaßnahmen selbst (oder auch die durch instabile Beschäftigungsverhältnisse verursachten Bedingungen) sind somit Untersuchungsgegenstände der vorausschauenden Gefährdungsbeurteilung und geraten über die Mitbestimmungsrechte des Betriebsrats im betrieblichen Arbeitsund Gesundheitsschutz in den Einflussbereich der Beschäftigten, womit sich neue Abwehrstrategien für Belegschaften und Betriebsräte eröffnen.

11 Kieselbach, T. et al. (2009): Gesundheit und Restrukturierung: Innovative Ansätze und Politikempfehlungen, München/Mering, S. 126.

\title{
Finanzmarktkrise und Verkaufsdruck im Finanzdienstleistungssektor
}

\author{
Herbert Bludau-Hoffmann, Roman Eberle \\ Klaus Holz-Skibinski, Uwe Spadzinski
}

\section{ARBEITSBEDINGUNGEN DER BESCHÄFTIGTEN IN DEN BANKEN}

Der Finanzdienstleistungssektor ist durch die Krise in Verruf geraten und bietet aufgrund der dort langjährig üblichen Verkaufspraktiken aktuell eine breite Angriffsfläche für Kritik - nicht nur für krisengeschädigte Kunden. Dabei haben viele Faktoren in der Arbeitsorganisation und in den Arbeitsbedingungen der dortigen Beschäftigten schon lange vorher alarmierende Warnzeichen gegeben.

Der Finanzdienstleistungssektor ist seit einigen Jahren geprägt von ehrgeizigen Wachstums- und Renditezielen, deren Ausmaß in den jeweiligen Häusern bisher unbekannt war. Ursächlich dafür ist für viele der internationale und oft finanzmarktgetriebene Wettbewerb, der die Renditeerwartungen von Ratingagenturen und Analysten in die Höhe treiben ließ. Diese Entwicklung führte letztendlich zu einem massiven Strukturwandel in den Finanzdienstleistungen - der sich vor allem durch eine deutliche Ökonomisierung aller Bearbeitungsprozesse bei den Banken auszeichnete. Die Beschäftigten sind seither direkter mit den wettbewerbsbedingt hohen Anforderungen des Marktes konfrontiert. Über vorgegebene Ertragsziele ist einer breiten Beschäftigtengruppe ein hohes Maß an Verantwortung für das erreichte Arbeitsergebnis übertragen worden, ohne dass sich dadurch ihre Entscheidungs- und Gestaltungsspielräume vergrößert hätten.

Dass sich dieser Strukturwandel auf die Arbeitsbedingungen der Beschäftigten niederschlägt, liegt auf der Hand. Das Selbstverständnis im Berufsbild des „Bankers" hat sich seither deutlich verändert. Die Beschäftigten müssen nun neben den üblichen (Beratungs-)Aufgaben vor allem als unternehmerisch Denkende und Handelnde agieren. Von ihnen wird in bisher ungekanntem Maße verlangt, „Profit zu machen " und sich selbst zu rentieren. Zwar hatten die Beschäftigten auch früher schon im Sinne des Unternehmens gewinnbringend tätig zu sein - sie hatten sich aber nicht in dem Maße um konkrete Umsatzsteigerungen ihres Unternehmensbereichs sowie aktive Kundenansprache zu kümmern.

\section{NEUE STEUERUNGSFORMEN IN DEN BANKEN}

Zur besseren Nutzung der Potenziale und der Kreativität der Beschäftigten setzt das Management in den Privatbanken, Genossenschaftsbanken und Sparkassen zunehmend auf neue Steuerungsformen, ${ }^{1}$ bei denen nicht die Leistung, sondern das Ergebnis der erreichten Arbeit im Vordergrund steht. Unter diesen neuen Steuerungsformen lassen sich vielfältige Arbeitsformen subsumieren: Mittlerweile

\footnotetext{
1 Peters, K./Sauer, D. (2005): Indirekte Steuerung - eine neue Herrschaftsform, in: Wagner, H.: Rentier ich mich noch?, Hamburg; Peters, K. (2001) Die neue Autonomie in der Arbeit, in: Glißmann, W./Peters, K.: Mehr Druck durch mehr Freiheit, Hamburg.
}

Herbert Bludau-Hoffmann, ver.di Essen.
Arbeitsschwerpunkt: Finanzdienstleistungen.
e-mail: herbert.bludau-hoffmann@ verdi.de
Roman Eberle, ver.di Dortmund.
Arbeitsschwerpunkt: Finanzdienstleistungen.
e-mail: roman.eberle@verdi.de
Klaus Holz-Skibinski, ver.di Bochum-Herne.
Arbeitsschwerpunkt: Finanzdienstleistungen.
e-mail: klaus.holz-skibinski@verdi.de
Uwe Spadzinski, ver.di Dortmund.
Arbeitsschwerpunkt: Finanzdienstleistungen.
e-mail: uwe.spadzinski@verdi.de


verbreitet (gerade im Bereich der Banken und Versicherungen) sind Zielvorgaben, Profit-Center oder internes Benchmarking. Gemein ist diesen Steuerungsformen, dass das Management selbst als unmittelbarer Weisungsgeber in den Hintergrund rückt und stattdessen die Beschäftigten in die Verantwortung für die unternehmerischen Ziele genommen werden.

Die Rahmenbedingungen bei diesen neuen Steuerungsformen sind typischerweise so ausgerichtet, dass die Belegschaften und die einzelnen Beschäftigten sich herausgefordert fühlen und mit Höchstleistung reagieren. Typisch sind beispielsweise kennziffernorientierte Systeme zum Ergebniscontrolling sowie die Herstellung von Konkurrenz sowohl zwischen einzelnen Beschäftigten als auch zwischen Geschäftsbereichen. Im Gegenzug werden Markterfolge und Ergebnissteigerungen mithilfe von Bonussystemen honoriert.

\section{WIRKUNGEN DER NEUEN STEUERUNGSFORMEN}

Da die Erreichung der Zielvorgaben für die Beschäftigten zwar schwierig, aber alternativlos erscheint, entscheiden sie sich dafür, alles für die Erreichung von Projekt- und Unternehmenszielen zu tun. Dabei werden persönliche Ziele - aber auch die eigene Gesundheit, die durch dauerhaften Stress in erheblichen Maß gefährdet wird - vernachlässigt. Solche Prozesse laufen auch ohne direkte Einflussnahme und das aktive Zutun der Vorgesetzten in den Teams selbst ab.

\section{MACHT BANK KRANK?}

Die Deutsche Angestellten-Krankenkasse (DAK) hat 2008 eine Gesundheitsbilanz für das Kreditgewerbe veröffentlicht. ${ }^{2}$ Die Studie wertet eine Befragung von mehr als 2.000 Bankangestellten aus. Die Ergebnisse zeigen, dass psychische Belastungen und Erkrankungen der Beschäftigten deutlich angestiegen sind. Als Gründe dafür werden Ängste vor weiterem Personalabbau, häufige Reorganisationen und Leistungsverdichtung gesehen. Die PARGEMA/WSI-Betriebsrätebefragung ${ }^{3}$ 2008/2009 hat gezeigt, dass der wachsende Arbeitsdruck in den Betrieben für die Finanzdienstleistungsbranche sogar überdurchschnittlich hoch ausfällt. Dabei werden insbesondere der Termin- und Zeit- druck, hoher Verantwortungsdruck und zu hohes Arbeitsvolumen als Belastungen wahrgenommen.

Zu bemerken ist, dass die Finanzmarktkrise die Probleme für Bankbeschäftigte sogar noch verschärft, da die Geschäftsmodelle und die Zielvorgaben trotz Wegbrechen der Märkte und Vertrauensverlust der Kunden nicht angepasst wurden.

\section{WIE GEHT ES WEITER?}

In der öffentlichen Diskussion ist man sich weitgehend einig darüber, dass verfehlte Leistungsanreizsysteme für das Bankmanagement und überzogene Renditeansprüche mit ursächlich für die Finanzkrise waren. Mittlerweile wurden auf gesellschaftlicher und politischer Ebene vielfältige Änderungsvorschläge gemacht, um eine Vertiefung oder gar eine Wiederholung der Finanzmarktkrise zu vermeiden. Dabei werden die Praktiken der Vertriebssteuerung im Finanzdienstleistungssektor ausgesprochen kritisch diskutiert. Leider steht der mittels Vertriebssteuerung erzeugte Verkaufsdruck der Beschäftigten weniger im Fokus. So werden heute für Fehl- und Falschberatungen zwar primär die Finanzdienstleistungsinstitute, aber auch deren Beraterinnen und Berater persönlich verantwortlich gemacht.

Inzwischen zeichnet sich $\mathrm{ab}$, dass die Banken zu einer Intensivierungsstrategie neigen anstelle von selbstkritischer Prüfung der verhängnisvollen Kennziffernund Zielvorgabensteuerung, der daraus resultierenden Abläufe und psychisch belastenden Reportsysteme, der Anreizstrukturen durch die Zahlung von Boni und damit einhergehender Verschärfung des Leistungsdrucks. Durch öffentliche Gelder abgesichert, strukturieren die Geldhäuser ihre Geschäfte um, jedoch nicht zur Entlastung der Vorgaben für Beschäftigte. Tendenziell stehen die Zeichen eher auf Abbau von Beschäftigung, d.h. die Arbeit wird künftig auf weniger Schultern verteilt.

Gewerkschaftliche Aufgabe muss es daher sein, sich schützend vor die Angestellten in der Branche zu stellen - und dabei in der Öffentlichkeit auch auf die belastenden Rahmenbedingungen, unter denen die Beschäftigten in den Instituten arbeiten, zu verweisen. Diese Arbeitsbedingungen in den Banken müssen deutlich humaner gestaltet werden.

\section{EINE VER.DI-KAMPAGNE FÜR BANKENBESCHÄFTIGTE}

Auf gewerkschaftlicher Ebene hat sich ver.di dieser Idee angenommen und im Rahmen eines groß angelegten Abstimmungsverfahrens im Dezember 2009 eine Reihe von Forderungen entwickelt. Die Forderungen lauten:

- Aussetzen der Zielvorgaben für dieses Jahr;

- Überprüfung aller Leistungsanreizsysteme;

- keine weitere Variabilisierung der Gehälter;

- kein ständiges Ändern von Vertriebsstrategien (Aktionen);

- Schluss mit den unwürdigen Praktiken des Vertriebscontrollings;

- kundenbedarfsgerechte Beratung statt Verkaufen auf „Teufel komm raus“;

- Finanzprodukte, die von Beratenden und Kunden verstanden werden.

Die Beschäftigten im Vertrieb von privaten Banken, Genossenschaftsbanken und Sparkassen waren aufgerufen, über diese Forderungen von ver.di abzustimmen. Die meiste Zustimmung erfuhren die Forderungen „Kundenbedarfsgerechte Beratung statt Verkauf „auf Teufel komm raus" und „Schluss mit unwürdigen Praktiken des Vertriebscontrollings“. Die hohe Zahl der Rückläufe bestätigt den enormen Druck, der auf den Beschäftigten im Vertrieb lastet.

Die Ergebnisse zeigen, dass die Beschäftigten in den Finanzdienstleistungen die Zusammenhänge zwischen den ökonomisierten Arbeitsbedingungen und dem oft krankmachenden Arbeitsdruck zunehmend erkennen. Eine Unterstützung der Beschäftigten, wie sie die ver.di-Kampagne im Fachbereich Finanzdienstleistungen seit einigen Jahren anbietet, könnte ein guter Ansatz sein, um die Arbeitsbedingungen im (krisenbedingt in Verruf geratenen) Finanzdienstleistungssektor zu verbessern und den Banken wieder ein menschliches Gesicht zu geben.

2 DAK-Forschung (Hrsg.) (2008): GesundheitsBilanz Kreditgewerbe, Arbeitsbedingungen und Krankenstand in Banken und Finanzinstituten, erstellt durch IGES-Institut, Hamburg. 